\title{
Propiedades psicométricas de la traducción al español de la Escala de Vinculación Prenatal Materna ${ }^{1}$
}

\section{Psychometric properties of the Mexican version of the Maternal Antenatal Attachment Scale}

\author{
Ruth Castro Mata ${ }^{2}$ \\ Universidad Autónoma de Nuevo León, México \\ Benito Estrada Aranda ${ }^{3}$ \\ Universidad Autónoma de San Luis Potosí, México \\ Rene Landero Hernández ${ }^{4}$ \\ Universidad Autónoma de Nuevo León, México
}

(Rec: noviembre 2014 - Acept: junio 2015)

\begin{abstract}
Resumen
El embarazo es el período de desarrollo y crecimiento óptimo del feto; paralelamente, para la madre, es un tiempo de compleja adaptación, en el cual además de experimentar cambios físicos, hay una mayor conciencia de su hijo, lo cual la aproxima afectivamente a este. El lazo que se establece entre la madre y el feto ayuda a mitigar los problemas físicos y emocionales de esta etapa, preparando la futura relación de apego entre madre e hijo. El objetivo del estudio es evaluar las propiedades psicométricas de la traducción al español de la Escala de Vinculación Prenatal Materna. Se utilizó el proceso de traducción inversa, a fin de lograr una equivalencia conceptual de la escala. El estudio incluyó a 169 mujeres embarazadas; a las cuales se aplicó la traducción de la escala de vinculación prenatal materna. El análisis factorial exploratorio mostró dos factores con el 36.8\% de la varianza explicada, eliminando 5 ítems. La escala total obtuvo un alfa de Cronbach de .694, la subescala de Calidad un alfa .771 y la de Preocupación de .550.
\end{abstract}

Palabras clave: vinculación prenatal maternal, propiedades psicométricas, apego.

\begin{abstract}
Pregnancy is the period for fetal development and growth. In parallel, for the mother is also a complex adaptation time, which in addition to experience physical changes, there is a greater awareness about their child that creates an affectively approach to his or her. The bond between mother and fetus helps to mitigate physical and emotional problems in this stage, preparing them for the future relationship between mother and child attachment. The objective in this study is to evaluate the psychometric properties of the Spanish translation of the MAAS. Reverse translation process was used in order to achieve a conceptual equivalence of the scale. The study included 169 pregnant women attending prenatal care. Exploratory factor analysis indicated two factors with $36.8 \%$ of the explained variance, along with 5 items that were removed. The total scale showed a Cronbach's alpha of 0.694, Quality subscale obtained an alpha of .771 and .550 Preocupation subscale.
\end{abstract}

Keywords: prenatal maternal vinculation, psicometric properties, attachment.

\footnotetext{
${ }_{1}$ Los autores desean agradecer al Hospital Regional Materno Infantil de Alta Especialidad de Guadalupe, Nuevo León, México, por el apoyo prestado para el acceso a la muestra.

2 Correspondencia a: Ruth Castro Mata. Universidad Autónoma de Nuevo León, UANL, Facultad de Psicología. Esquina Mutualismo 110 Mitras Centro, C.P. 64020, Monterrey, México. Universitaria San Nicolás de los Garza Nuevo León, C.P. 66451 México. Tel. (81) 83294050. E-mail: eunice_36@hotmail.com.

3 E-mail: benito.estrada@uaslp.mx.

4 E-mail: rlandero1_mx@yahoo.com.mx.
} 


\section{Introducción}

La idea de que existe un vínculo afectivo entre la madre y el feto durante la gestación no es un tema nuevo; en efecto, desde mediados del siglo XX se plantearon nociones de cómo la mujer establece una conexión con el feto de manera intrapsíquica, y que la carga libidinal de la mujer embarazada es transmitida hacia el bebé en gestación (Benedek, 1959; Bibrin, 1959; Deutch, 1945; Winnicott, 1958).

John Bowlby (1969), fue uno de los pioneros en contribuir al tema de vinculación-apego. Su trabajo original se centró en la necesidad biológica del infante de experimentar una cercanía o proximidad física con la madre, complementando posteriormente, que la vinculación es también una necesidad afectiva y que va más allá de procesos inconscientes. A partir de los trabajos de Bowlby, se desarrollaron investigaciones más profundas respecto al apego y vinculación. Una de las investigadoras que profundizó en el tema fue Mary Ainsworth, la cual sostiene que la vinculación del infante es más allá que un proceso biológico, es decir, pues incluye la apreciación interna del comportamiento materno (Ainsworth, Blehar, Waters \& Wall, 1978).

La importancia de establecer una buena vinculación prenatal, radica en que se da la posibilidad de establecer relaciones más apropiadas, después del nacimiento, las que serán la base para un apego seguro padres-hijo, vínculo necesario para el desarrollo del niño (Smeekens et al., 2007).

La vinculación temprana surge de la experiencia recíproca e interactiva entre el bebé y las figuras parentales y el mecanismo de apego innato, el cual se puede entender como un movimiento recíproco en el que bebé y madre, incitan y modifican al otro a través de la interacción de señales y conductas que se producen de forma sincronizada desde la gestación (Enríquez, Padilla \& Montilla, 2008).

Hay una relación perceptiva-psicológica, la cual concede al niño la oportunidad de establecer comunicación con la madre, permitiéndole asimilar los estados emocionales de esta, así como su actitud vivencial hacia él; estas relaciones son la base para el establecimiento de un vínculo temprano (Valencia \& González, 2008).

Partiendo de que la vinculación afectiva prenatal se concibe como un lazo o vínculo emocional que normalmente se desarrolla entre la mujer embarazada y su aún no nacido infante (Condon, 1993), esta podría ser una valiosa herramienta desde la cual la madre expectante podría valerse para hacer más llevaderos los problemas físicos y emocionales que sufre directa y/o indirectamente durante esta etapa (Rodrigues, PérezLópez \& Brito, 2004).

Se ha encontrado que las representaciones que crea la mujer embarazada acerca del bebé tienen que ver con esperanzas, expectativas, temores y deseos sobre este, al mismo tiempo que tienen que ver consigo misma como madre y con su propia historia de apego (Ammanti, 1992; Grimal \& Heresi, 2012; Hesse, 1996).

La vinculación prenatal que establece la madre con su hijo por nacer se hacen evidentes mediante representaciones mentales que tienen lugar durante el embarazo, tales como la presencia o ausencia de afecto, el tipo de narrativa respecto a la descripción de ella misma, su bebé, pareja y hasta su propia madre, coherencia en la narrativa de sus emociones e ideas, flexibilidad para recibir nueva información y aceptar nuevas alternativas (Ilicali \& Fisek, 2004).

La mayoría de las investigaciones acerca de la vinculación prenatal materna hacen uso de escalas de autoreporte con el fin de medir este lazo entre la madre y el feto. Entre los instrumentos más utilizados se encuentra el Fetal Attachment Scale (FAS) (Cranley, 1981), el Inventario de Apego Prenatal (PAI) desarrollado por Muller (1994), y la Escala de Vinculación Prenatal Materna (Condon, 1993). Cada uno de ellos ha sido evaluado encontrando fortalezas y debilidades (Van den Bergh \& Simons, 2009).

El instrumento desarrollado por Condon (1993) llamado Escala de Vinculación Prenatal Materna, permite estudiar el desarrollo del apego materno-fetal sin que las variables como el temperamento del niño y el ambiente postnatal influyan, de este modo, se enfoca casi exclusivamente en pensamientos y sentimientos acerca del bebé por parte de la madre, complementando así otros instrumentos, que a juicio de Condon, se centraban sólo en las actitudes de la madres respecto a estar embarazada y su rol materno.

En este contexto, la herramienta permite en la madre, evaluar las conductas concretas como el deseo de interactuar con el feto, la elaboración imaginativa de características del bebé, y el placer o satisfacción derivadas de estas actividades, sin involucrar las actitudes hacia el embarazo o hacia el rol de la maternidad.

El instrumento de Condon y Corkindale (1997), mide dos factores de la vinculación; el primer factor, "Calidad" que evalúa las emociones y pensamientos positivos con respecto a la representación interna del bebé, cercanía, ternura, el deseo de conocer y ver a su bebé. El segundo factor es "Intensidad de Preocupación", que mide la preocupación de la madre hacia el bebé, incluyendo la cantidad de tiempo que pasa pensando 
y hablando acerca de él, evaluando de esta manera el lugar que el feto ocupa en la vida emocional de la mujer.

La escala de Vinculación Prenatal Materna, clasifica la vinculación en cuatro cuadrantes (Figura 1) en donde cada uno representa un estilo: "fuerte/saludable", "afecto positivo/baja preocupación", "desvinculado/ ambivalente", y "ansioso, ambivalente o preocupación sin afecto".

El Cuadrante 1, Calidad positiva y Preocupación alta (fuerte/saludable), se refiere a las mujeres altamente preocupadas por el feto, cuya intensidad de preocupación es acompañada por sentimientos de cercanía, ternura y deseo por su bebé.

El Cuadrante 2, Calidad positiva y Preocupación baja (afecto positivo/baja preocupación), ubica a aquellas madres que reportan afectos positivos de vinculación (similares a los del cuadrante 1). Sin embargo, pasan menos tiempo desarrollando la experiencia de vinculación. En este cuadrante se podrían ubicar las madres multíparas ya que para ellas los cambios ya son conocidos y los toman como normal; del mismo modo, se pueden ubicar las mujeres que perciben la intensidad de la experiencia de vinculación como amenazante (ej.: Temor de perder el bebé).

El Cuadrante 3, Calidad negativa y Preocupación baja (desvinculado/ambivalente) se refiere a las mujeres que pasan poco tiempo pensando en el feto y experimentan pocos afectos positivos asociados a la vinculación. Puede ser que se sientan separadas del feto y debido a eso están poco interesadas por él, o pueden tener sentimientos ambivalentes hacia el feto los cuales son amenazantes por lo que su existencia es suprimida o negada.

\section{CALIDAD DE VINCULACIÓN}

(positiva)

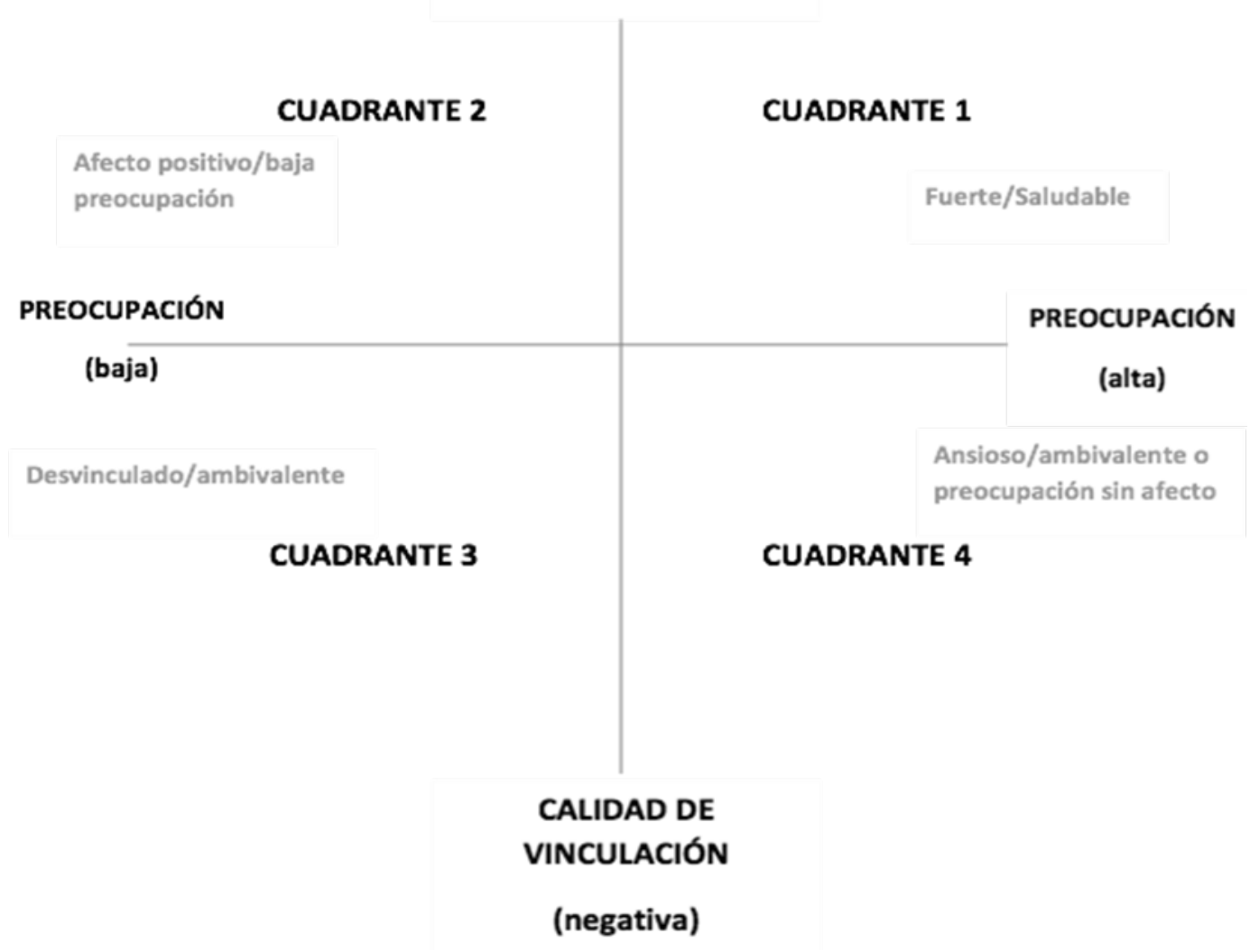

Figura 1. Cuadrantes de la escala de Vinculación Prenatal Materna. 
El Cuadrante 4, Calidad negativa y Preocupación alta (ansioso, ambivalente o preocupación sin afecto), ubica a aquellas mujeres que se preocupan demasiado por el feto, sin embargo, la preocupación es sin afecto o acompañada de ambivalencia. En ambos casos, está desprovisto de matices afectivos placenteros o positivos. Puede tener un toque de interés intelectual por el feto. Cabe señalar, que los sentimientos ambivalentes pueden generar algún tipo de preocupación ansiosa (ej.: madres preocupadas por el bebé o por sí misma, que excluyen de la experiencia los aspectos positivos de la vinculación).

El objetivo del presente estudio es examinar las propiedades psicométricas de la traducción al español de dicha escala para poder contar con un instrumento adecuado que mida la vinculación prenatal materna en población mexicana.

\section{Método}

\section{Participantes}

La muestra quedó conformada por mujeres embarazadas que asistieron a consulta de atención prenatal del Hospital Regional Materno-Infantil de alta especialidad, del municipio de Guadalupe, Nuevo León, México, las cuales aceptaron voluntariamente a participar en la investigación. De esta manera, se constituye una muestra no probabilística de tipo intencional, de 169 participantes.

Dentro de las características de la muestra se encuentra la edad media de 23.24 años $(\mathrm{DE}=5.24)$, siendo la edad mínima 13 y la máxima 41 años. Por otra parte, todas las mujeres se encontraban en el tercer trimestre de gestación; el 60\% reportaron haber completado su educación hasta nivel secundaria, el $38.3 \%$ hasta bachillerato, mientras que solo el $1.7 \%$ cursó una licenciatura; el $83.3 \%$ de la muestra reportó dedicarse exclusivamente al hogar y el $3.3 \%$ se encontraba con trabajo y en periodo de incapacidad por el embarazo, mientras que el $13.4 \%$ reportó ser estudiante.

Asimismo, el 56.7\% de las mujeres reportó haber planeado su embarazo, mientras que el $43.3 \%$ no lo planificó; el $93.2 \%$ no experimentó ninguna complicación a lo largo de la gestación, mientras que el $6.8 \%$ reportó haber presentado algún tipo de complicación. En otra característica, 35\% de las mujeres eran primíparas, $36.7 \%$ tenían solo un hijo, $18.3 \%$ dos hijos y $10 \% 3$ hijos.
En cuanto a la relación de pareja, el $43.3 \%$ reportó que su relación era mejor que antes del embarazo, el $31.7 \%$ igual que antes, $8.3 \%$ con problemas ocasionales y el $3.7 \%$ consideraba su relación peor que antes del embarazo; el $13.3 \%$ de la adolescentes no contaba con pareja. Un $21.7 \%$ de las adolescentes reportó haberse sentido deprimida por un periodo mayor a 15 días en algún momento de su vida, mientras que el $78.3 \%$ restante no ha experimentado sentimientos depresivos.

\section{Instrumentos}

1. Cuestionario socio-demográfico: se diseñó y aplicó un cuestionario socio-demográfico, con el propósito de tomar en consideración alguna de las variables, así como para seleccionar a las participantes que cumplieran con los criterios de inclusión establecidos previamente.

2. Escala de Vinculación Prenatal Materna (Maternal Antenatal Attachment Scale, MAAS): fue diseñada por Condon (1993), como un cuestionario de auto reporte conformada por 19 ítems. Las respuestas son ubicadas en 5 puntos de una escala tipo Likert, la puntuación mínima es 19 y la máxima 95.

Las puntuaciones en la escala de Calidad puntúan entre 11 y 50, mientras que en la de Preocupación, entre 8 y 40 . La escala total cuenta con una consistencia interna alfa de Cronbach de .818 .

Se clasificó Calidad y Preocupación como positivo o alto, según fuera el caso, cuando la puntuación se encontraba por arriba de la media del grupo, y como negativo o bajo, respectivamente cuando puntuaba por debajo de la media.

Debido a que este instrumento solo se encontraba en idioma inglés, se llevó a cabo el procedimiento de traducción inversa planteado por Cohen y Jones (1990), cuyo proceso es descrito por Masson (2005). Cohen y Jones plantean este enfoque de traducción como "comparativo", ya que el resultado pretende hacer referencia a los constructos del instrumento a través de las culturas.

Cuando la traducción del inglés original se hace palabra por palabra (lingüísticamente equivalente) puede llegar a perderse el significado de la idea tal cual como se quiere transmitir. Es por eso, que se propone como objetivo principal el lograr una equivalencia conceptual, donde los conceptos expresados en español sean equivalentes al significado original escrito en la versión en inglés (Varrichio, 2004). El resultado es una traducción simétrica que supone ser familiar y coloquial al mismo tiempo (Cohen \& Jones, 1990). Se requiere 
una re-evaluación de las propiedades psicométricas para el instrumento resultante de la traducción.

A fin de lograr una equivalencia conceptual en la traducción del idioma inglés al idioma español, Masson (2005) recomienda un proceso de traducción y traducción inversa por parte de un equipo formado por lo menos de 2 personas bilingües. Basados en dicha recomendación el equipo se compuso de las siguientes personas:

Persona 1: maestra del idioma inglés cuya lengua natal es el español.

Persona 2: persona cuya lengua natal es el idioma inglés, pero domina el español de manera fluida.

Persona 3: Investigador, con conocimientos avanzados del idioma inglés cuya lengua natal es el español.

Una vez que la persona tres comparó la equivalencia de la traducción inversa con la original, y constató que eran homogéneas lingüísticamente, se procedió a utilizar la versión traducida al español.

\section{Procedimiento}

El presente estudio es parte de una investigación de tipo no experimental, longitudinal de panel, de tipo descriptivo, sobre roles sexuales, vinculación afectiva prenatal y depresión pre y postparto (llevada a cabo de mayo del 2010 a junio del 2012).

Haciendo uso del paquete estadístico SPSS 18, se efectuaron los análisis correspondientes; realizando análisis descriptivos de las características de la muestra mediante las opciones de cuartiles, media, desviación estándar, mediana, valores mínimos y máximos, frecuencias y porcentajes; además, se hizo uso de tablas de contingencia con el fin de identificar los estilos de vinculación. También se calculó el índice de fiabilidad del instrumento y sus subescalas mediante el coeficiente alfa de Cronbach, obteniéndose correlaciones entre las subescalas usando el coeficiente de correlación de Pearson. Para medir la validez del instrumento se empleó el análisis factorial exploratorio con el método de extracción de componentes principales y Varimax en rotación, y así determinar el número de factores siguiendo el procedimiento utilizado por el autor de la escala original.

\section{Resultados}

Los estadísticos descriptivos de la escala se muestran en la tabla 1. Se obtuvo una confiabilidad alfa de Cronbach para la escala total de .77, la subescala de Calidad un alfa de Cronbach de .76, y la de Preocupación de .51. Asimismo, se encontró una correlación de Pearson significativa y positiva entre las subescalas de Calidad y Preocupación( $\mathrm{r}=.397$, $\mathrm{p}$ $=.000)$.

Tabla 1

Estadísticos descriptivos de la escala MAAS

\begin{tabular}{ccccc}
\hline & Media & D.E. & Min. & Max. \\
\hline Total MAAS & 75.83 & 9.2 & 50 & 95 \\
Calidad & 43.44 & 5.7 & 24 & 50 \\
Preocupación & 28.11 & 4.8 & 17 & 40 \\
\hline
\end{tabular}

El $62.1 \%$ de la muestra tuvo un nivel de Calidad de vinculación alto o positivo, mientras que el $37.9 \%$ nivel bajo o negativo; por su parte la subescala de Intensidad de Preocupación tuvo un $57.4 \%$ bajo y un $42.6 \%$ alto. La frecuencia de los estilos de vinculación prenatal propuestos por Condon (1993) se presenta en la tabla 2, siendo el estilo Fuerte/Saludable el de mayor porcentaje y el Ansioso/Ambivalente el de menor presencia.

Tabla 2

Estilos de Vinculación Prenatal Materna

\begin{tabular}{ccc}
\hline Estilos de vinculación prenatal & Frecuencia & Porcentaje \\
\hline $\begin{array}{c}\text { C1. Fuerte/saludable } \\
\text { C2. Afecto positivo/baja } \\
\text { preocupación }\end{array}$ & 56 & 33.1 \\
$\begin{array}{c}\text { C3. Desvinculado/ambivalente } \\
\text { C4. Ansioso/ambivalente o } \\
\text { preocupación sin afecto }\end{array}$ & 49 & 29.0 \\
Total & 16 & 9.5 \\
\hline
\end{tabular}

Se empleó el análisis factorial exploratorio, mediante el método de reducción de componentes principales se obtuvieron seis factores, los cuales explicaban el $58 \%$ de la varianza. Se redujeron aquellos factores que representaban varianza residual, confirmándolo a su vez con el gráfico de sedimentación por lo que se efectuó el análisis factorial exploratorio con dos factores como lo plantea el autor en el estudio original, dando una varianza explicada de $36.8 \%$, con una medida de adecuación Kaiser-Mayer-Oklin (KMO) de .749.

Se eliminaron los ítems uno y seis debido a su carga factorial baja, así como los ítems 11, 13 y 19 ya que compartían varianza en los dos factores. La escala 
quedó conformada por dos factores: el primero con nueve ítems que corresponde a subescala de "Calidad" (ítems $3,5,7,9,10,12,15,16,18$ ); y el segundo con cinco ítems correspondiente a la subescala de "Intensidad de la Preocupación" (ítems 2, 4, 8, 14 y 17).

Tanto el ítem cinco (.388) como el ocho (.396) obtuvieron cargas factoriales ligeramente menores a 40 . Únicamente los ítems cinco y 18 cargaron en un factor diferente a la escala original (preocupación), marcando en el de Calidad. Asimismo, el factor siete que en el original no cargó en ninguno de los dos factores, se ubicó satisfactoriamente en el factor de Calidad. La escala total obtuvo un alfa de Cronbach de .694, la subescala de Calidad un alfa .771 y la de Preocupación de .550.

\section{Discusión}

En el presente estudio se evaluaron las propiedades psicométricas de la versión mexicana en español de la escala de Vinculación Materna Prenatal (Condon, 1993). Se encontró que la escala global y la subescala de Calidad cuentan con una adecuada consistencia interna; sin embargo, la subescala de Preocupación presentó una baja consistencia interna. En cuanto a la escala global, se encontró una ligera y menor diferencia en el alfa de Cronbach que el reportado por Condon (1993) en su estudio original. Pese a ello, la consistencia interna encontrada en el presente estudio de acuerdo con los criterios de Nunnally y Bernstein (1994) es adecuada (Alfa de Cronbach $\geq .70$ ).

En cuanto al alfa de Cronbach de las subescalas, se encontraron diferencias con lo reportado por Van Bussel, Spitz y Demyttenaere (2010), donde la subescala de Calidad obtuvo un menor alfa y la de Preocupación una puntuación mayor que la obtenida en esta investigación. No obstante, contaron con una adecuada consistencia interna, a diferencia de lo encontrado en el presente estudio en donde la escala de Preocupación no alcanza una aceptable consistencia interna (alfa de Cronbach $\leq .70$ ).

Entre las subescalas de Calidad y Preocupación se encontró aunque significativa y positiva una débil correlación a diferencia de lo encontrado en otros estudios (Condon \& Corkindale, 1997; Priel \& Besser, 2001; Van Bussel, Spitz \& Demyttenaere, 2010).

Asimismo, tanto la media de la escala global como de las subescalas de nuestro estudio se asemeja a las obtenidas originalmente por Condon (1993) y las encontradas en otras investigaciones (Condon \&
Corkindale, 1997; Hart \& McMacho, 2006; Van Bussel, Spitz \& Demyttenaere, 2010).

Hasta donde sabemos, no todos los estudios toman como referencia esta clasificación propuesta por Condon (1993) de los estilos de vinculación, los cuales se obtienen usando las medias de las subescalas como punto de corte. Cabe señalar, que se encontró un estudio (Van Bussel, Spitz \& Demyttenaere, 2010) que sí reporta la frecuencia de estos y en donde se encontraron resultados similares a los expuestos en la presente investigación, vale decir, mayor frecuencia en el estilo fuerte/saludable y menor frecuencia en el ansioso/ambivalente. Sin embargo, debido a la débil correlación entre las subescalas (Calidad y Preocupación) y la baja consistencia interna de la subescala de Preocupación, existe la duda de que esta clasificación realmente represente los estilos de vinculación entre la madre y el feto, por lo que se sugiere una interpretación cautelosa de estos estilos.

Al revisar la teoría en que se basan las subescalas, encontramos que puede haber confusión en cuanto a la interpretación de los ítems, pues si bien las dos subescalas se refieren a los pensamientos que tiene la madre, la de Calidad se centra en los pensamientos positivos y la de Preocupación en la cantidad de tiempo que piensa en el feto; entonces, se cree que las mujeres pudieran enfocarse solo en la calidad de los pensamientos, ya que se observó cómo la mayoría de los ítems se agrupan en esta subescala.

Se obtuvo una validez de constructo mediante el análisis factorial exploratorio, en el cual la prueba KMO fue adecuada, indicando así un índice de correlación genérico entre todos los ítems. Condon sugiere que la escala se divide en dos subescalas: Calidad de la vinculación e Intensidad de la Preocupación (o tiempo dedicado a vincularse). En la presente versión en español se pone en duda la subescala de Preocupación ya que a diferencia de la original que agrupa ocho ítems, en esta solo se agruparon cinco ítems, además de reportar una baja confiabilidad tanto en el análisis con los ítems originales, como después de la reducción de factores.

Por su parte, la subescala de Calidad se agrupó de manera similar a la versión original, con la diferencia de que agrupó los ítems cinco y dieciocho, originalmente correspondían a la subescala de Preocupación, explicándose esto porque no le otorgan tanta importancia al tiempo que pasan imaginándose al bebé, si no a los pensamientos o emociones que experimentan y el nivel de cercanía que establecen con el bebé. Asimismo, un dato importante es que el ítem siete, el cual en la escala original no se agrupó en ninguno de los dos factores, cargó satisfactoriamente en la subescala de Calidad 
entendiendo así que, para las mujeres mexicanas, sentir que el bebé es dependiente de ellas genera un sentimiento de cercanía.

Se eliminaron cinco ítems de la escala; cuatro de ellos correspondían a la subescala de Calidad y solo uno a la de Preocupación.

En la versión traducida se observan la distribución de los ítems en dos factores (gráfica de sedimentación), tal como lo sugiere Catell (1966). De esta manera el factor uno se agrupa nuevamente como la subescala de Calidad y el factor dos la subescala de Preocupación de acuerdo con lo planteado por Condon (1993).

La disparidad entre los resultados se pueden explicar por la diferencia entre la cultura australiana y mexicana. La mujer mexicana, por un lado, le da mayor importancia a la calidad de la vinculación contemplando las emociones y pensamientos positivos o negativos con respecto a la representación interna que se tiene del bebé, cercanía, ternura y deseo de conocer y ver a su hijo. Por otro lado, le resta importancia a la preocupación que experimenta hacia el bebé, lo que se traduce en el menor tiempo -e intensidad-que le dedica a conocer sobre el desarrollo intrauterino.

Hasta donde conocemos, no se cuenta con datos en población mexicana con los cuales se puedan contrastar los datos encontrados. Una investigación realizada en Chile (Ossa, Bustos \& Fernández, 2012) utilizó esta escala; sin embargo, no expone las propiedades psicométricas encontradas, ni cómo se realizó la traducción del instrumento. Por lo anterior, se recomienda realizar nuevos estudios utilizando el análisis factorial confirmatorio para poder corroborar los datos y llegar a un consenso para la utilización de esta escala, corroborando la reducción y distribución de los ítems.

\section{Referencias}

Ainsworth, M., Blehar, M., Waters, E. \& Wall, S. (1978). Patterns of attachment: A psychological study of the Strange Situation. Hillsdale: Erlbaum.

Benedek, T. (1959). Parenthood as a developmental phase: A contribution to the libido theory. Journal of the American Psychoanalytic Association, 7, 389-417. doi:10.1177/000306515900700301

Bowlby, J. (1969). Attachment and Loss Volume I: Attachment. New York: Basic Books.

Cattell, R. (1966). The scree test for the number of factors. Multivariate Behavioral Research, 1(2), 245-276. doi:10.1207/ s15327906mbr0102_10

Condon, J. \& Corkindale, C. (1997). The correlates of antenatal attachment in pregnant women. British Journal of Medical Psychology, 70(4), 359-372. doi:10.1111/j.2044-8341.1997.tb01912.x

Condon, J. (1993). The assessment of antenatal emotional attachment: Development of a questionnaire instrument. British Journal of Me- dical Psychology, 66(2), 167-183. doi:10.1111/j.2044-8341.1993. tb01739.x

Cranley, M. (1981). Development of a tool for the measurement of maternal attachment during pregnancy. Nursing Research, 30(5), 281-284. Recuperado de http://journals.lww.com/nursingresearchonline/Abstract/1981/09000/Development_of_a_Tool_for_the Measurement_of.8.aspx

Cohen, H. \& Jones, E. (1990). Interpreting for cross-cultural research. Changing written English to American Sign Language. Journal of the American Deafness \& Rehabilitation association, 24(2), 41-48. Recuperado de http://psycnet.apa.org/psycinfo/1992-29645-001

Deutch, H. (1945). The psychology of women. New York: Grune \& Stratton.

Enríquez, A., Padilla, P. \& Montilla, I. (2008). Apego, vinculación temprana y psicopatología en la primera infancia. Informaciones psiquiátricas, 3(193), 249-292. Recuperado de http://www.revistahospitalarias.org/info_2008/03_193_03.htm

Hart, R. \& McMahon, C. (2006). Mood state and psychological adjustment to pregnancy. Archives of Women's Mental Health, 9(6), 329-337. doi:10.1007/s00737-006-0141-0

Hesse, E. (1996). Discourse, Memory, And The Adult Attachment Interview: A Note With Emphasis On The Emerging Cannot Classify Category. Infant Mental Health Journal, 17(1), 4-11. doi:10.1002/ (SICI) 1097-0355(199621)17:1<4::AID-IMHJ1>3.0.CO;2-S

Ilicali, E. \& Fisek, G. (2004). Maternal representations during pregnancy and early motherhood. Infant Mental Health Journal, 25(1), 16-27. doi:10.1002/imhj.10082

Masson, T. (2005). Cross-cultural instrument translation: Assessment, translation and statistical applications. American Annals of the Deaf, 150(1), 67-72. doi:10.1353/aad.2005.0020

Nunnally, J. \& Bernstein, I. (1994). Psychometric theory. New York: McGraw Hill.

Ossa, X., Bustos, L. \& Fernandez, L. (2012). Prenatal attachment and associated factors during the third trimester of pregnancy in Temuco, Chile. Midwifery, 28(5), e689-e696. doi:10.1016/j. midw.2011.08.015

Priel, B. \& Besser, A. (1999). Vulnerability to postpartum depressive symptomatology: dependency, self-criticism and the moderating of antenatal attachment. Journal of Social and Clinical Psychology, 18(2), 240-253. doi:10.1521/jscp.1999.18.2.240

Rodrigues, A., Pérez L. \& Brito N. (2004). La vinculación afectiva prenatal y la ansiedad durante los últimos tres meses del embarazo en las madres y padres tempranos. Un estudio preliminar. Anales de psicología, 20(1), 95-102. Recuperado de http://www.um.es/ analesps/v20/v20_1/09-20_1.pdf

Smeekens, S., Riksen-Walraven, J. \& Van Bakel, H. (2007). Multiple Determinants of Externalizing Behavior in 5-Year-Olds: A Longitudinal Model. Journal of Abnormal Child Psychology, 35(3), 347-361. doi:10.1007/s10802-006-9095-y

Valencia, M. \& González, W. (2008). Etología del apego y del reconocimiento en el ser humano. El Hombre y la Máquina, 31, 40-51. Recuperado de http://www.redalyc.org/articulo.oa?id=47803104

Van den Bergh, B. \& Simons, A. (2009). A review of scales to measure the mother-foetus relationship. Journal of Reproductive and Infant Psychology, 27(2), 114-126. doi:10.1080/02646830802007480

Van Bussel, J., Spitz, B. \& Demyttenaere, K. (2010). Reliability and validity of the Dutch versión of the maternal antenatal attachment scale. Archives of Women's Mental Health, 13(3), 267-277. doi:10.1007/s00737-009-0127-9

Varrichio, C. (2004). Measurement issues concerning linguistic translations. En M. Frank-Stromborg \& S. Olsen (Eds.), Instruments for clinical health-care research (pp.55-64). Masschusetts: Jones and Barlett.

Winnicott, D. (1958). Collected Papers: Through pediatrics to psychoanalysis. New York: Basic Book. 
